\title{
LA TORMENTA PERFECTA QUE ACABÓ CON EL CHANCHO: CAMBIOS EN LA PERCEPCIÓN DE LA LIMPIEZA EN LOS ANDES RURALES
}

\author{
THE PERFECT STORM THAT ENDED THE PIG: CHANGES IN THE \\ PERCEPTION OF CLEANLINESS IN THE RURAL ANDES
}

\author{
Jordi Gascón ${ }^{1}$
}

\begin{abstract}
La sociedad rural e indígena tiene una concepción de la limpieza diferente a la urbana y criolla, aunque la relación de subordinación entre ellas hace que las segundas transfieran sus valores a las primeras. A partir de un caso de estudio (la desaparición del chancho en la Isla de Amantaní, Lago Titicaca), planteamos que esta transferencia tiene dos aspectos. Por un lado, una transformación en la concepción de la dualidad pureza-contaminación. Este cambio en la mentalidad es resultado de diferentes vectores que adoctrinan al indígena (escuela, turismo, servicio militar...) o inoculan nuevos valores por contagio (emigración, doble residencia...). El otro aspecto viene determinado por cambios en el contexto económico y en las estrategias de reproducción, que permiten sustituir prácticas que ahora están en desacuerdo con la nueva concepción de limpieza por otras nuevas. La desaparición del chancho y los cambios en la concepción de la limpieza que lo explican son un microcosmos que refleja las relaciones de dependencia y subordinación del mundo rural e indígena frente al urbano y occidental, las estrategias de los isleños para articularse a esta sociedad, y las diferencias sociales y económicas que hay entre ellos.
\end{abstract}

Palabras claves: limpieza, higiene, población indígena, habitus, hecho social total, turismo.

Rural and indigenous society has a different conception of cleanliness to that of urban and white creole society. However, the relationship of subordination between them causes the latter to transfer their values to the former. Analyzing a case study (disappearance of the pig on Amantani Island, Lake Titicaca), we propose that this transfer comprises two aspects: on the one hand, there is a transformation in the conception of the purity-pollution duality. This change in mentality is the result of different vectors, some aimed at indoctrinating the native (school, tourism, military service...), and others at grafting on them new values by way of transmission (migration, double residence...). The second aspect is determined by changes in the economic context and reproduction strategies. These changes allow for the replacement of practices, now discordant with the new conception of cleanliness, by new ones. The disappearance of the pig and the changes in notions of hygiene that can explain it are a microcosm: one that reflects the relationships of dependency and subordination, distinguishing not only the rural and indigenous world from the urban and western world, but also the strategies of the islanders to adapt to this society, and the social and economic differences that exist between them.

Key words: Cleanliness, hygiene, indigenous peoples, habitus, total social factor, tourism.

Hasta la década de 2000, el cerdo o chancho (Sus scrofa domestica) era un animal omnipresente en Amantaní, la isla más grande que el Perú tiene en el lago Titicaca, habitada por población quechua. Todos los grupos domésticos criaban, al menos, una pareja de cerdo criollo. Normalmente cada año tenían una camada que, o bien se vendía, o bien se utilizaba para sustituir a los progenitores cuando estos dejaban de tener capacidad reproductiva o eran vendidos o sacrificados. El chancho de Amantaní era un animal de tamaño pequeño y de pelaje oscuro. Comparado a las especies mejoradas, el rendimiento del cerdo criollo es bajo en términos de reproducción y crecimiento, pero no requiere muchos insumos (Ramos 2008): en Amantaní, su alimentación se basaba en lo que encontraba en el campo, en subproductos agrarios y en una sopa espesa preparada con restos de la cocina en la que predominaba la monda de la papa. Su crianza tampoco exigía grandes esfuerzos: vivía en el traspatio de las casas, y vagaba con libertad por áreas y caminos colindantes. Llevarlos a los pastizales era trabajo de los miembros más jóvenes del grupo doméstico.

A finales de la década del 2000, el número de familias que criaban chanchos estaba disminuyendo. En la segunda mitad de la década siguiente el animal

1 Universitat de Barcelona, Observatori de l’Alimentació (ODELA), Barcelona, Cataluña, España. jordigascon@ub.edu

Recibido: noviembre 2019. Aceptado: julio 2020.

http://dx.doi.org/10.4067/S0717-73562021005002002. Publicado en línea: 6-octubre-2021. 
desapareció de la isla. Por esa misma época, la mayor parte de las familias dejaron de criar cuy, o lo aislaron en jaulas situadas en el traspatio. Hasta entonces, el cuy o coballa (Cavia porcellus), que se alimentaba también de restos agrarios y de la comida familiar, nunca había salido de la cocina, espacio en el que se movía libremente.

El objetivo del presente artículo no es tanto conocer qué le sucedió al chancho y al cuy, como observar, a través de este fenómeno, los cambios en la percepción de la limpieza, la higiene y la pureza de la población rural andina, y detectar los factores que explican estos cambios. Hay que recordar que conceptos antónimos como pureza y contaminación, o higiene e insalubridad, son relativos. La frontera se establece simbólicamente. Son convenciones socialmente aceptadas dentro de un determinado esquema de valores que determinan qué se debe hacer y qué no, y sufren transformaciones con el tiempo (Moser 2002; Smith 2007; Reno 2015). Siguiendo la senda estructural-simbólica iniciada por Mary Douglas (2003 [1966]), la antropología ha analizado los cambios en los sistemas de clasificación de lo que se entiende por limpieza. Sin embargo, no ha sido un tema especialmente estudiado en el mundo andino ${ }^{1}$, aunque la mirada urbana, "blanca" y racista que identifica indígena con suciedad ha sido ampliamente acreditada (p.ej., Kingman y Bretón 2017; Orlove 1998; Pérez et al. 2014).

Creemos que la desaparición del chancho y la marginación del cuy fue resultado de dos tipos de factores: los que impulsaron un cambio en la percepción de la limpieza y la suciedad, que analizamos en la segunda sección del artículo; y otros, de carácter material e instrumental, que han permitido transformar las prácticas para adecuarse a esa nueva percepción, y que veremos en la tercera sección. El presente trabajo plantea la hipótesis de que los cambios en la concepción de las dicotomías pureza-contaminación, o higiene-insalubridad, no solo son resultado de un cambio de mentalidad, sino también de oportunidad; es decir, de la disponibilidad de determinados recursos que permiten abandonar unas praxis y sustituirlas por otras nuevas.

\section{Marco Teórico}

El devenir del chancho y del cuy en un distrito rural andino puede tener poco valor en sí mismo. El interés radica en que cristaliza una realidad social, económica, religiosa y política compleja. En otras palabras, la desaparición del chancho y del cuy se puede considerar como lo que Marcel Mauss (2009 [1925]) denominó un hecho social total (fait social total): un acontecimiento concreto que pone de manifiesto el orden global en el que se circunscribe. Es una parte que nos remite al todo. En nuestro caso, el papel del chancho y del cuy en cada momento histórico nos permite observar la situación de subordinación de la población indígena y rural.

Un hecho social total no alude necesariamente a una estructura inmutable, tal como lo concibió Claude Lévi-Strauss (1950). Un hecho social total puede ser síntoma de cambios (Cazeneuve 1968; Gurvitch 1990 [1964]). Como veremos, la nueva noción de limpieza y pureza que empieza a arraigar en la sociedad amantaneña en las últimas décadas del siglo pasado, y que llevará a la desaparición del chancho, no solo fue resultado de la influencia de una sociedad occidental y urbana hegemónica, sino también de las estrategias que cada familia adoptó para articularse a una realidad cambiante en el que la isla y sus habitantes cada vez se encontraban menos aislados.

Por tanto, aunque la determinación de lo que es limpio y sucio parece establecido, en el cotidiano, por reglas naturales inmutables, en realidad se trata de una convención culturalmente creada. Los códigos de pureza e higiene ilustran el concepto bourdieano de habitus. Es decir, la de un principio generador de representaciones y prácticas sociales: representaciones a través de los cuales el individuo percibe la realidad, y prácticas que le permiten actuar de una forma considerada como razonable y coherente por el grupo sin ser necesariamente consciente de ello (Bourdieu 1980).

Como toda convención social, como todo habitus, los códigos de limpieza son producto de la historia y se transforman con el tiempo. Bourdieu identifica dos factores que impulsan los cambios en el habitus. El primero, la necesidad de ajustarse a un contexto cambiante. Unas normas y prácticas obsoletas, desactualizadas a las variaciones contextuales, pueden dificultar la adaptación de la sociedad a la sociedad mayor e incrementar su vulnerabilidad. Pero esta transformación se puede hacer de diversas maneras. El elemento que marca cómo se hace la transformación son las relaciones sociales, mediadas por el capital económico, social y cultural acumulado por cada individuo o grupo social (Bourdieu 1979).

En el caso que nos ocupa, veremos como la transformación en el sentido de la limpieza, el aseo y la pureza es resultado, por un lado, de una mayor 
interrelación del microcosmos amantaneño con la sociedad mayor; en cierta manera, es el resultado de la consolidación del estado-nación. En este sentido, como decíamos, esa transformación materializa las relaciones de dominación y subordinación entre el mundo rural-indígena y el urbano-occidental. Pero Amantaní no es un sujeto paciente, ni tampoco está conformada por una sociedad homogénea.

Tal como lo define Bourdieu, el habitus limita lo que es socialmente aceptable y adecuado. Pero, por un lado, dentro de esos límites ofrece libertad de maniobra al individuo. Y por otro, esos límites pueden sufrir transformaciones, creando nuevas prácticas. Eso es lo que hace que el habitus no sea un mecanismo automático: las decisiones de cada individuo es resultado de un cálculo estratégico, no necesariamente consciente, en el que pone en juego su capital social, económico, educativo y cultural. En Amantaní, aquellos isleños que acumulaban mayor capital simbólico y económico fueron imponiendo nuevas prácticas higiénicas que permitían una mejor articulación con la sociedad mayor y que, a la postre, se acabaron convirtiendo en las consideradas apropiadas y correctas. En la medida que no todos tuvieron la misma capacidad (capital) para asumirlas, tampoco todos pudieron aprovechar de la misma manera las nuevas formas de articulación con la sociedad mayor. Por ejemplo, determinados sectores quedaron marginados de nuevas fuentes de ingresos como el turismo, y entre el resto, la distribución de los beneficios fue desigual.

\section{Metodología y Unidad de Análisis}

Con 9,28 km², Amantaní (Departamento de Puno, Perú) es la isla más grande del Lago Titicaca. Su población, alrededor de 3.500 habitantes, son todos indígenas quechuas (Instituto Nacional de Estadística e Informática, INEI 2018). Zona tradicionalmente agropecuaria y pesquera, en la actualidad su economía está más diversificada. Junto con la isla de Taquile, las islas Uros y algunas comunidades de la vecina península de Capachica, es uno de los destinos de los tours que visitan el Titicaca partiendo de la ciudad de Puno, la capital departamental. Buena parte de la población ha vivido la emigración o practica la doble residencia entre la isla y Puno, Juliaca o alguna otra ciudad más distante. El fortalecimiento presupuestario de las instituciones públicas distritales, y el establecimiento e incremento de los servicios educativos y sanitarios en las últimas dos décadas, han supuesto también nuevas fuentes de ingresos de carácter burocrático-funcionarial. A pesar del surgimiento de estas nuevas fuentes de ingresos, toda la población sigue dedicándose a la producción agraria, pero debido al incremento demográfico y al sistema de herencia divisa, las tierras que cada grupo doméstico posee son insuficientes para mantener su economía.

La metodología aplicada ha sido la etnográfica, dirigida a conocer y comprender el comportamiento social de una determinada población utilizando técnicas de investigación cualitativas: la observación participativa y la realización de entrevistas semiestructuradas. Este método permite analizar el discurso de los individuos en relación a su contexto social, histórico e ideológico. En Amantaní venimos realizando investigación etnográfica desde 1990, si bien la mayor parte de la información utilizada para el presente trabajo se obtuvo entre 1990 y 1995, y en una reciente estancia de tres meses, entre junio y septiembre de 2019.

\section{Factores que Hicieron Desaparecer al Chancho (1): Cambio en la Percepción de la Limpieza}

\section{El chancho molesta. Los discursos emic}

Con el turismo, las casas se han modernizado. No hay lugar para los cuyes (V 2016).

(Los chanchos) huelen un poco. Ya no se quieren a lado de la casa (O 2016).

Cuando se pregunta a los isleños por qué dejaron de criar chanchos y cuyes, predomina un discurso negativo hacia la convivencia con los animales. Las citas anteriores ilustran las dos principales justificaciones. La primera apunta al cambio en la estructura del hogar. Hasta la década de 1980, la vivienda tradicional consistía en dos pequeños edificios de una sola planta, construidos de adobe y techo de totora y paja, con pequeñas ventanas sin cristal cerradas con un postigo de madera. Ninguno de los dos edificios estaba compartimentado. Uno servía de habitación para toda la familia. El otro, normalmente algo más pequeño y bajo, hacía el doble papel de cocina y comedor. El suelo de la cocina, de tierra compactada, y la estufa, de arcilla y encendida prácticamente todo el día, era un buen ecosistema 
para los cuyes que, como hemos explicado, corrían libremente por ese espacio. En la década de 1980 y 1990, las cocinas empezaron a construirse más grandes, altas y con el suelo cimentado. En la década siguiente el tamaño siguió incrementándose y aparecieron los primeros fogones de gas, que solo se encendían para cocinar. La cocina, ahora más fría, dejó de ser un buen ecosistema para los cuyes.

El chancho también dejó de tener espacio en las nuevas estructuras habitacionales. En la década de 1980 aparecieron las primeras casas de dos pisos, ahora compartimentadas en diferentes habitaciones, con ventanas acristaladas y tejados de calamina. Las nuevas viviendas se construían con tres cuerpos que rodeaban un patio central. Detrás de estos edificios se situaban el corral ovino y la porqueriza. En las últimas tres décadas, el incremento de la población y del tamaño de las residencias ha acentuado la densidad de espacio construido en los núcleos poblacionales ${ }^{2}$. Encontrar un lugar adecuado para colocar la porqueriza sin molestar a los habitantes de la casa ni a los vecinos empezó a ser complicado.

$Y$ es que el chancho, como se indica en la segunda cita, ahora olía. Un nuevo sentido de la limpieza lo empezó a considerar un animal sucio que debía distanciarse del espacio de vivienda. Algunos isleños también explican la desaparición del chancho por la triquinosis. "Tenía chancho, pero con triquina, y hubo que botarlo. Por gusto criamos dos años" (J, 2019). La afección real del parásito no parece haber sido muy importante; pocos isleños recuerdan haber tenido algún animal que lo padeciera. Pero es un discurso que incrementa la percepción de impureza.

No son las únicas razones que los isleños dan para explicar la desaparición del cerdo. Se aduce, también, que comportaba mucho trabajo. Hemos visto que, inicialmente, el esfuerzo destinado a su cría no era muy elevado. Pero a medida que la nueva sensibilidad sobre la higiene se fue afianzando, aumentó la necesidad de limpiar periódicamente la porqueriza y de controlar que el animal no invadiera el terreno del vecino. Además, la concentración urbanística, que redujo el espacio en el que podían moverse con libertad y encontrar comida cerca del caserío, obligó a llevarlo a zonas de pasto con mayor frecuencia.

\section{El factor turístico}

En Amantaní, donde más hemos trabajado es en (las comunidades de) Occopampa y en Occosuyo, porque sus líderes han organizado a cinco, a nueve familias, en su comunidad, en donde ellos, aparte de ofrecer la alimentación y el hospedaje, que las camas estén limpias y adecuadas, hacen una presentación de una actividad cultural (...). Yo fui al $80 \%$ de las viviendas de Amantaní, a explicar qué hay, qué falta. Algunos han dicho "sí, señora". Otros se han molestado (VQ, gerente de agencia de viajes, 2019).

A finales de la década de 2000, Guillaume Perche (2012) analizó las consecuencias del turismo en la estructura del hogar en Amantaní. Este autor explicaba que este sector económico había colonizado los espacios privados a través de la reconfiguración de la casa y de las pautas de higiene. Aunque atribuir únicamente al turismo los cambios en la percepción de la limpieza es exagerado, no se puede negar que es uno de los factores causales más importantes.

El turismo no es una actividad nueva en Amantaní. A finales de la década de 1970 los isleños, animados por el éxito de la vecina isla de Taquile, destino turístico de las rutas que pasaban por el Titicaca, iniciaron su promoción. El turismo se consideró un recurso comunal; es decir, de usufructo exclusivo para los isleños. Se estableció que el alojamiento y alimentación se realizaría en las casas particulares, para lo que debían tener al menos una habitación acondicionada. Igualmente, solo las embarcaciones de Amantaní podían transportar a los visitantes. La legislación sobre derechos indígenas permitió estas prerrogativas.

La dificultad de acceso a la isla (solo había unas pocas lanchas disponibles para trasladar a los viajeros, y de escasa potencia) hizo que los primeros años no fueran muy productivos. Y cuando el número de visitantes empezó a incrementarse, el conflicto armado entre Sendero Luminoso y las fuerzas armadas hundió el turismo en el Perú. La recuperación del sector en el país iniciado a mediados de la década de 1990, una vez limitado el conflicto armado a zonas marginales de la Amazonía, incrementó el número de visitantes en Amantaní. El surgimiento de nuevas lanchas más rápidas facilitó un fuerte crecimiento. No hay datos fiables sobre el volumen de turistas que recibe la isla. El Ministerio de Turismo afirma que en 2017 fueron de 37.336 (MINCETUR 2019a), pero es un 
dato que se basa solo en información recogida por la Capitanía de Guardacostas Lacustre de Puno ${ }^{3}$. Un número importante de las lanchas que salen de la ciudad de Puno no son contabilizadas por la Capitanía, y ninguna de las que trasladan visitantes desde la vecina península de Capachica. El número real es sustancialmente superior.

Que el turismo funcione como un recurso comunal no implica que sus beneficios se distribuyan equitativamente ${ }^{4}$. Actualmente algo más de la mitad de la población recibe beneficios del turismo, si bien una minoría, con contactos directos con agencias de viajes de Puno, Cusco e incluso Arequipa o Lima, son los que más aprovechan este recurso. Los cambios estructurales en la vivienda isleña se explican, en parte, por la exigencia de confort e higiene reclamados por el turismo, así como por los ingresos que esta actividad proporcionó a quienes obtenían sus beneficios y que les permitió invertir capital en su construcción, mejora o ampliación.

Pero ahora nuestro interés se centra en el papel de las agencias de viajes. El carácter de recurso comunal del turismo tampoco implica que los isleños tengan pleno control sobre el sector. De hecho, son dependientes de estas agencias, que proveen de viajeros a la isla. $Y$ este predominio les permite no solo negociar precios, sino también condiciones. La cita que inicia el subapartado, extracto de una entrevista realizada a una gerente de una de las agencias de viaje que operan con Amantaní, expone una de esas condiciones: las pautas de limpieza han de ser las apropiadas, entendiendo como tales las urbanas y occidentales.

En la primera mitad de la década de 2010, la Municipalidad Distrital de Amantaní negoció con diversas agencias puneñas distribuir a sus clientes siguiendo un sistema de rotación. Actualmente algo más de la mitad de los isleños reciben turistas mediante este sistema. No obstante, el reparto de los beneficios sigue siendo muy disímil. Aquellos con una infraestructura más preparada tienen acuerdos bilaterales con las agencias de viaje más exigentes, y la incursión reciente de páginas web como Booking también está generando cambios que facilitan la articulación de esta minoría con el mercado turístico.

Si bien el sistema de rotación permitió incrementar el número de población beneficiada por el turismo, también comportó una mayor exigencia en relación a la calidad. La Municipalidad, temerosa que las agencias que participan en la rotación abandonen el proyecto si sus clientes se quejaban del servicio, incorporó nuevos mecanismos de supervisión. Por ejemplo, a inicios de 2019 se estableció el cargo de presidente de la Comisión de Turismo, contratado por la Municipalidad. Este cargo tiene dos objetivos: defender los intereses de los isleños que alojan turistas frente a las agencias de viajes, y mejorar la calidad del servicio e infraestructuras turísticas. En relación a este segundo objetivo, dos campañas ocuparon los esfuerzos del presidente en sus primeros meses en el cargo: concienciación sobre la limpieza y gestión de la basura, y atención al turista. Además, indirectamente se encarga de controlar la calidad del servicio: "(Los alojamientos) tienen que tener su baño, una cama buena, la cocina... y que todo esté limpio y ordenado. ¿Y eso lo controla alguien? No, no hay nadie. Ahora lo estoy haciendo yo" (A, presidente de la Comisión de Turismo, 2019).

Para asegurar la viabilidad del sistema de rotación, la Municipalidad se ha convertido en garante de las condiciones de higiene y calidad del servicio. Conociendo sus limitaciones legales para imponer determinadas condiciones, en julio de 2019 esta institución solicitó a la Dirección de Turismo de Puno, organismo dependiente del gobierno departamental, que realizase controles de calidad de los alojamientos, con gran disgusto de la población. Estos controles se iniciaron en agosto. El presidente de la Comisión de Turismo y el alcalde municipal preveían que un número indefinido de alojamientos ("los que no se esfuerzan y bajan el nivel de la isla") serían clausurados.

Por tanto, las mismas instituciones públicas de Amantaní aceptaban que el porcentaje de la población que no aloja turistas (entre un 40 y un $45 \%$ de las familias isleñas a finales de 2019) se incrementaría con estas medidas en favor de la calidad. Una parte de los grupos domésticos que no participan en la actividad turística está conformada por isleños que pasan la mayor parte del año en la emigración, o que tienen otras fuentes de ingresos bien remunerados (propietarios de tiendas de abarrotes, maestros constructores, etc.). Pero cuando se pregunta a los isleños, identifican dos perfiles como los más característicos. Uno son las familias que no tienen suficiente capital, ya sea porque están conformadas por parejas recién formadas que aún no han tenido tiempo para ahorrar y arreglar convenientemente sus casas, o bien porque su pobreza es de carácter estructural.

Hay otras familias que no reciben porque no están en condiciones. Ellos mismos se 
califican. Otros dicen "Tener turismo es una pérdida de tiempo, con tiempo hay que hacer las cosas, la atención, preparar la cama..... Es que ahora, solo el colchón, vale soles. ¡un simple colchón! Es por eso que a veces otras familias no están en condiciones (L 2019).

Pero el perfil más característico es de los ancianos, a los que se acusa de tener casas viejas no acondicionadas, y especialmente, un sentido de la higiene no adecuado. Cuando las comunidades organizan el reparto de turistas a través del sistema de rotación, muchos guías y agencias de viaje se oponen a que sus clientes vayan a las casas de los más mayores, y esta actitud recibe la comprensión y la connivencia del resto de comuneros. El resultado es que no suelen alojar más de la mitad de los turistas que le toca al resto, o incluso menos. La misma Municipalidad, en su deseo de contentar al sector empresarial en sus requerimientos de calidad e higiene, acentúa el proceso que los expulsa del negocio:

Estamos viendo que esas personas ya mayores tienen que dedicarse a otro tipo de actividad. A hacer música, o a una presentación cultural de su tejido, como la bayeta. Ese tipo de cosas... taller de piedra. Este tipo de demostraciones. Ese plan lo estamos viendo. Porque ya están un poco cansaditos, pero como necesitan, quieren ser parte del sistema de rotación (M, alcalde distrital de Amantaní, 2019).

De hecho, se ha disparado una carrera por mejorar las infraestructuras privadas de alojamiento, en las que prima el concepto de nuevo como sinónimo de higiénico, para poder establecer o mantener acuerdos bilaterales con agencias de viajes y obtener una mayor parte de los beneficios totales del turismo... aunque ello obliga a reinvertir buena parte de los ingresos obtenidos. En los dos últimos años, por ejemplo, algunas familias han iniciado la construcción de habitaciones con baño privado. A medida que se acelera este círculo vicioso, la exigencia de calidad aumenta, y la lógica urbana y occidental de higiene se consolida.

\section{Disciplinamiento}

El servicio militar era duro, fuerte pegaban, pero buenas cosas he aprendido.
Era una especie de escuela, donde había un respeto de mayor a menor, y donde aprendí a hablar delante de público, sin timidez. Y muchas otras cosas. Por eso me ha gustado bastante. Había que tener higiene: andar limpiecito, no estar sucio, tener limpia la cama... A algunos esto no les gustaba. No les gustaba lavarse (AV 1995).

El turismo ha sido un vector de adoctrinamiento en educación sobre higiene, pero no ha sido el único ni el primero. Diferentes estrategias de control social sobre la población indígena se basan en la asunción de los valores del sector social dominante (Fanon 2015 [1952]); entre ellos, los de la limpieza y la gestión del cuerpo.

La cita con la que hemos iniciado el subapartado hace referencia a una de estas estrategias: el servicio militar. El ejército es una institución dirigida no solo a la defensa del territorio o la vigilancia armada de la población, sino también a facilitar un proceso de integración nacional que pasa por "civilizar" al indígena (Toche 2005). El servicio militar dejó de ser obligatorio en Perú en 1999, pero previamente los isleños habían tenido cierta habilidad para sortear la conscripción militar, así que pocos han pasado por el cuartel. Pero esta minoría, una vez licenciada y retornada a Amantaní, trasmitía los valores inculcados. Y lo hacía con éxito: gracias al estatus social que otorgaba ser reservista, se convertían en líderes de opinión y ejemplos a seguir. Incrementaba, además, las posibilidades de asumir cargos políticos de relevancia, desde los cuales podían difundir determinados principios. El caso de AV, del que es la anterior cita, es ejemplar: a principios de la década de 1990 asumió el que entonces era el principal cargo distrital, el de gobernador, y a principios de la década siguiente, el de alcalde, cuando la Municipalidad ya se había convertido en la institución política más importante.

La escolarización también ha jugado un papel civilizatorio destacable (Degregori 2003). Amantaní cuenta con una escuela de secundaria y diversas de primaria, en las que son ubicuos los afiches sobre limpieza y aseo personal. Periódicamente instituciones de salud y alguna ONG realizan campañas sobre salubridad e higiene, que complementan el trabajo más cotidiano de los docentes, todos foráneos de origen urbano, en estas materias.

Todos los centros educativos son fiscales, excepto uno de confesión adventista. Es la escuela más antigua que tiene la isla, lo que indica el arraigo histórico de 
esta religión. La primera misión de la Iglesia Adventista del Séptimo Día en la región se estableció en el pueblo de Platería, en la orilla aymara del Titicaca, en 1910, y al poco tiempo algunos isleños ya habían asumido el nuevo culto como estrategia para alfabetizarse (Gascón 2005a, 2017). Con el tiempo surgirían otras congregaciones en Amantaní, como el Movimiento de los Santos de los Últimos Días (mormones), que tuvo actividad entre las décadas de 1980 y 2010, o más recientemente, la Misión Israelita del Nuevo Pacto Universal (israelitas). Todas ellas propugnan una nueva concepción de la pureza que pasa por no ingerir alcohol, y dejar de pijchar (mascado de coca) y de comer carne de animales considerados impuros, como el cerdo y el cuy. Muestran, además, una especial preocupación por la higiene personal y la limpieza de la ropa. Acusando de ello a la opresión gamonal, el que fuera primer evangelizador adventista en la zona del Titicaca en la década de 1910 afirmaba que:

\section{Los indígenas (vivían) en una condición verdaderamente deplorable. Vivían en la más abyecta miseria e ignorancia. Desconocían hasta las reglas más sencillas de higiene (...). No conocían cuchillos, tenedores o cucharas, y comían sus alimentos con los dedos sin lavar. Nunca se bañaban, ni lavaban sus vestidos (Stahl sf:85-86).}

Si bien el porcentaje de creyentes no católicos nunca ha sido grande, la mayoría ha sido, en algún momento y por un tiempo más o menos largo, adventista; en menor medida, mormón o israelita. Los principios propugnados por estas confesiones han ido impregnando el comportamiento cotidiano de la población, incluso de aquella que nunca desertó del catolicismo. Por ejemplo, fiestas particulares como las bodas, que tradicionalmente duraban tres días, se redujeron a dos o a uno, y con menos fastos. Las nuevas iglesias no católicas también repudian este tipo de celebraciones, que consideran un derroche inútil. Esta influencia también ha sido patente en el ámbito de la higiene y en la marginación del chancho. Volviendo al tema de los festejos, el asado de uno o varios chanchos enteros había sido, en tiempos, un elemento central de cualquier celebración particular. Mucho antes de la desaparición del cerdo en la isla ya había quedado marginado de las fiestas, ya fuera porque entre los convidados siempre había adventistas, ya porque otros productos alimentarios adquiridos en el moderno mercado convencional empezaron a tener un mayor papel como consumo posicional.

Finalmente, no se puede olvidar el papel de las campañas de salubridad estatales y de determinadas organizaciones no gubernamentales. Como hemos visto, muchas de ellas se canalizaban a través de las escuelas e iban dirigidas a los estudiantes y a sus madres. Otras llegaban directamente a los hogares, como las destinadas a incorporar las llamadas "cocinas económicas", más limpias y eficientes energéticamente. Pero estas acciones no parecen haber tenido un rol determinante en el cambio de la percepción de la higiene, ya que fueron siempre escasas y puntuales.

\section{Urbanización}

La emigración ha sido una constante en la historia de Amantaní desde la década de 1940. La isla había sido, desde la Colonia, tierra de haciendas. Las relaciones sociales de producción se caracterizaban por extraer al colono su trabajo y producción excedente. A cambio, el sistema proveía los mecanismos que posibilitaban la reproducción campesina. Pero tras un largo periodo de luchas y conflictos que se remontan al último tercio del siglo XIX, en esa década se inició un proceso de compra de las haciendas por parte de sus colonos. Este proceso terminó en 1964, con la venta de la más grande ${ }^{5}$. La necesidad de obtener el dinero necesario para la adquisición de los fundos hizo que su población saliese a buscar trabajos temporales.

Lo que fue una actividad con un objetivo puntual, terminó siendo una práctica crónica. El crecimiento demográfico, que en un sistema de herencia divisa hizo disminuir los recursos agrícolas per cápita, los bajos precios de los productos agrarios en el mercado convencional, o el mayor acceso a este mercado y a su variada oferta de productos industriales de uso cotidiano, incrementó la necesidad de obtener unos ingresos que no se podían conseguir en la isla. En los años 1960 y 1970, la emigración de los jóvenes se convirtió en un rito de paso, tras lo cual volvían con el capital necesario para formar una familia y los materiales para construir una nueva casa; muchos ya no regresaban. A partir de 1980, la emigración temporal, durante unos meses al año o incluso varios años, era una práctica habitual.

La mejora de los sistemas de transporte marítimo y vial (lanchas más rápidas y apertura de la ruta por la península de Capachicha) acortó la duración del 
viaje a las ciudades de Puno, capital administrativa del departamento, y Juliaca, el polo económico. Esto facilitó que, a partir de la década de 2000, un porcentaje de la población practicase la doble residencia: entre alguna de estas ciudades y la isla. El desarrollo del turismo impulsó estrategias de este tipo. Muchos empezaron a destinar el capital que obtenían fuera de Amantaní en la mejora de sus casas en la isla para conseguir ventajas competitivas en el nuevo sector económico. Y este desplazamiento de recursos no solo era de capital; también de perspectiva. Los gustos occidentales y urbanos, con los que esta población convivía e iba asimilando, empezó a influenciar en el diseño de las nuevas construcciones residenciales amantaneñas y en las nuevas lógicas sobre limpieza e higiene. Amantaní, sin dejar de ser rural, se urbanizó en su actitud.

El acceso a las nuevas tecnologías de la información y la comunicación, como la radio, la televisión, el celular o, más recientemente, internet, permite a los jóvenes amantaneños estar conectados con ese mundo urbano de gustos occidentales sin salir de la isla. Una conexión que influye en sus prácticas cotidianas, como la música, el vestuario o los hábitos de higiene.

Disciplinamiento mediante diversos mecanismos (turismo, escuela, religión...) e inoculación de unas costumbres urbanas y occidentales cada vez más cercanas a través de la emigración y las TIC, explican el cambio en la percepción de lo que es sucio y limpio, de lo que es puro y contaminado. Pero, difícilmente esta transformación hubiera sido posible sin un "racismo higiénico" (Colloredo-Mansfeld 1998) que identifica lo rural e indígena con suciedad. Una ideología que no solo existe en la estructura mental de los grupos sociales dominantes, sino que es asumida por la población indígena como una forma de colonización del pensamiento.

\section{Factores Que Hicieron Desaparecer al Chancho (2): Cambio en la Disponibilidad de Recursos}

\section{Para qué sirve un chancho}

Hubo otra ocasión en el que el ganado porcino disminuyó notablemente en Amantaní, si bien entonces quedó lejos de desaparecer. Fue cuando los campesinos tuvieron que acopiar dinero para comprar las haciendas, proceso del que ya hemos hablado. Comenzó una carrera por obtener la mayor cantidad posible de dinero, ya que las tierras que cada grupo doméstico percibiría en el reparto dependían de su aportación en la compra: "Los colonos juntaban plata vendiendo papa, vendiendo vaca, vendiendo chancho" (T 1991). Así, a medida que los fundos se vendían, los colonos se quedaban sin ganado.

Este periodo ya distante de la historia de la isla nos permite comprender que el papel del chancho, del cuy, y del ganado en general, en una economía campesina va más allá de su aportación alimentaria, o en tejido y cuero (Ríos Ocsa 1992). Criar cerdos y cuyes era una estrategia que permitía enfrentar la vulnerabilidad en contextos caracterizados por la dificultad de acceder a un mercado de bienes y de trabajo, por unos recursos agrarios limitados, y por los riesgos inherentes a la producción agraria, siempre dependiente del clima y los fenómenos meteorológicos. A eso hay que sumar el carácter parsimonioso de la agricultura: la cosecha no siempre está disponible ante a una emergencia. En estos casos (enfermedad, entierros, mala cosecha, etc.), el cerdo y el cuy eran un activo que se podía vender rápidamente, actuando como fondo de seguro. También servían como mecanismo de ahorro para enfrentar inversiones futuras, como los festejos que acompañan a un matrimonio, la asunción de cargos religiosos y políticos, que siempre comportan gastos ceremoniales, o el costo de escolarización de los hijos. Por su parte, el cuy permitía adquirir aquellos productos de consumo cotidiano que no se producían en la isla, como fósforos o material escolar.

A principios de la década de 1990 existía la memoria de que, en tiempos, la mayor parte del cerdo era consumido por la familia.

En aquellos tiempos buenos chanchos habían. (...) Y no vendía la carne en Puno. Todito traía para acá. Y se hacía en chicharrón. De tres chanchos, por decir, uno se hacía chicharrón; otro para dar a la familia; y el otro, enterito, para el dueño. Del que se hacía chicharrón se compartía también con la familia. Y manteca salía dos, tres latas. Esa manteca se vendía en Puno. Mi papá vendía y con eso compraba ropa, cuadernos, querosene... Eso hacía mi papá (AM 1992).

Pero al menos desde la década de 1980 , solo se consumía en la isla en ámbitos ceremoniales y festivos, como las bodas. Su uso alimentario más común era en forma de manteca y chicharrón; es decir, como insumo para la cocina. 
El chancho ofrecía otro beneficio: sus purines. Alimentado con restos que no tendrían otra utilidad, el resultado era un potente fertilizante que contiene macronutrientes primarios para la agricultura (nitrógeno, fósforo y potasio), así como otros secundarios y diversos micronutrientes. Este abono se destinaba a la chacra doméstica (espacio de producción agraria contiguo al caserío), que en su papel de hortus es cultivado de forma intensiva. A esa chacra también iban los restos orgánicos que se acumulaban en el suelo de la cocina y se barrían con regularidad, entre los que se encontraban los excrementos de los cuyes.

\section{Nuevas praxis}

La transformación de la percepción de la limpieza fue lento, generacional. También lo fue el surgimiento de nuevas prácticas que materializaban ese cambio en la mentalidad. Pero los procesos no fueron paralelos. Solo cuando los amantaneños tuvieron acceso a una serie de recursos, les fue posible activar nuevos hábitos y abandonar los, hasta ese momento, tradicionales.

En relación al chancho y al cuy, hay que entender que sus utilidades antes explicadas se daban en un determinado contexto: el de una sociedad históricamente aislada social y económicamente. Las relaciones de producción en las que el amantaneño vivía mientras funcionó el sistema de hacienda imponía este aislamiento. Esas relaciones, que le obligaban a trabajar las tierras de la hacienda y en otras labores personales para el hacendado, le enajenaba de su fuerza de trabajo excedentaria, de tal manera que no podía acceder al mercado laboral libre. Igualmente, el colono tampoco tenía acceso al mercado de bienes, ni como consumidor ni como proveedor, ya que el sistema le enajenaba también su producción excedente mediante diversas estrategias: obligación de comprar en el almacén de la hacienda, facilidad para el endeudamiento, asunción de obligaciones ceremoniales (cargos en el sistema tradicional de fiestas), etc. Finalmente, también existía un aislamiento coercitivo: explícitamente, el amantaneño tenía prohibido desplazarse fuera de la isla si no era por encargo del hacendado. De alguna manera, vivir en una isla era una metáfora de su aislamiento económico (Gascón 1999). La adquisición de la tierra por parte de la población isleña supuso su incorporación al mercado moderno laboral y de productos, pero siguió siendo una incorporación limitada. Las condiciones del mercado no le eran favorables: trabajos mal remunerados y precios agropecuarios bajos (Gascón 2005a).
Esta situación cambió a finales de la década de 1990. Diversos factores explican la mejora de la situación económica del isleño y el incremento de su capacidad adquisitiva. Por un lado, en la década del 2000 se superó el estado de crisis que había caracterizado las décadas anteriores. El crecimiento reflejado en las estadísticas macroeconómicas desde 1990 parecía haber llegado a los sectores populares; entre ellos, los rurales-andinos (Asensio 2017). Nuevos programas asistenciales gubernamentales proveen de un pequeño ingreso a los amantaneños en peor situación económica, convirtiéndoles también en consumidores. La Municipalidad Distrital, la máxima autoridad de la Isla, empezó a gestionar fondos públicos, muchos de los cuales se destinaron a la construcción o rehabilitación de infraestructuras en los que se emplea mano de obra isleña mediante el tradicional sistema del trabajo comunitario, pero que ahora se remunera. A ello hay que sumar el factor turismo. Solo en turismo internacional, entre 2004 y 2018, Perú pasó de recibir 1,4 a 4,4 millones de visitantes (MINCETUR 2019b). El Titicaca y sus islas, uno de los principales destinos del país, notaron este fuerte crecimiento, que se tradujo en un importante incremento de los ingresos. Finalmente, las mejoras en el transporte facilitaron una relación más fluida entre la ciudad y la isla, que permitió aplicar nuevas prácticas económicas como la doble residencia, de la que ya hemos hablado. Estas últimas décadas también se han caracterizado por un incremento demográfico de la población y de la urbanización de los núcleos poblados, que muchas veces no quedan reflejados en los censos poblacionales resultado de estas estrategias de doble residencia.

En este nuevo contexto fue desapareciendo la utilidad que tenían el chancho y el cuy. Por ejemplo, el incremento de los ingresos y el mayor acceso al mercado laboral moderno redujo el papel del ganado como mecanismo para enfrentar la vulnerabilidad campesina. La formación de un sistema de ahorro y crédito al que el campesino puede acceder, eliminó el papel que el ganado tenía como fondo de inversiones y urgencias. A esto hay que sumar la disminución de los gastos ceremoniales. El número de fiestas que se realizan en Amantaní es menor que el de antes, y la mayoría se sufragan con el apoyo de la Municipalidad y cuotas comunitarias; ha desaparecido la onerosa, y obligatoria, figura del alferado. Ahora, además, los principales cargos políticos están remunerados y reciben presupuestos estatales que les ha liberado de los gastos ceremoniales que antes comportaban. 
Desapareció el papel del chancho como fondo de ahorro para cubrir esos gastos.

El incremento poblacional y la herencia divisa han reducido la cantidad de tierras disponibles para cada familia, por lo que el papel del chancho como proveedor de abono ya no es tan necesario. Además, en los últimos años se ha establecido un mercado barato y accesible de estiércol con la península de Capachica, zona productora de ganado ovino y, en menor medida, de auquénido. Es común encontrar en los muelles grandes bolsas de este abono que los capachiqueños transportan a demanda de los isleños.

La mayor urbanización de los núcleos habitados, tanto por el crecimiento demográfico como por la construcción de viviendas más amplias con nuevas infraestructuras domésticas (baños, etc.), redujo el ecosistema habitual del chancho: la chacra doméstica y los terrenos entre caseríos. Es habitual recibir como respuesta a la pregunta por la desaparición del chancho, que los animales "sufrían" por esta razón. Como hemos visto, en el último periodo de su existencia en Amantaní, su crianza supuso un incremento del trabajo resultado de esa falta de espacio: ya no podían dejarse en libertad y había que llevarlos con asiduidad a las zonas de pasto.

Otro factor a tener en cuenta fue el crecimiento del porcentaje de población que alojaba turistas, y que, por lo tanto, estaban obligadas a mantener unas determinadas medidas higiénicas en sus hogares: esta población pasó de un 8,5\% a mediados de la década de 1990 (Gascón 2005a) a superar el 55\% un cuarto de siglo después.

Finalmente, su rol como insumo alimentario también desapareció. En la década de 1960, los amantaneños empezaron a recibir ayuda alimentaria de la USAID a través de Cáritas. Uno de los productos estrella era el aceite vegetal. Después de un periodo inicial de rechazo ("Nadie, nadie quería este aceite. Nadie. Nadie de la comunidad sabía cómo utilizarlo. Hasta los ratones se morían en el aceite, ¡cómo es líquido! Nadie quería”. AV, 1994), el aceite entró en el hábito alimentario del isleño y sustituyó el papel de la manteca y el chicharrón de cerdo.

Así, poco a poco, en el año 60 se empezó a usar. En el año 70, con más fuerza. Hoy lo utilizamos para cualquier cosa. Para freír, para aderezo... ya no se utiliza ni manteca ni chicharrón (AV 1994).

El nuevo hábito culinario arraigó de tal manera que, a finales de la década de 1990, cuando la ayuda alimentaria terminó, los amantaneños empezaron a comprarlo.

\section{Conclusiones}

No podemos comer carne de animales que no sean rumiantes. Sí podemos criar, pero para vender. Yo tengo cuyes, y mi hermano también tiene chanchos (H 1990).

Hemos analizado el caso del chancho y del cuy con una mirada que podríamos denominar "microetnográfica", adaptando el término de microhistoria; la microhistoria es la rama de la historia social que estudia casos particulares que, en sí mismo, tienen un interés marginal, pero que reflejan y permiten entender el funcionamiento de la estructura social. Y esto es posible porque la higiene y la salubridad se pueden interpretar como lo que Marcel Mauss (2009 [1925]) denomina un hecho social total; es decir, un fenómeno o práctica que refleja el orden global. La desaparición del chancho, ubicuo en el paisaje amantaneño hasta la década de 2010, y los cambios en el sentido de la limpieza y la higiene que lo explican, son un pequeño universo que sintetiza las relaciones de dependencia y subordinación del mundo rural e indígena frente al urbano y occidental, las estrategias de los isleños para articularse a esta sociedad, y las diferencias sociales y económicas que hay entre ellos.

A diferencia de como lo conceptualiza el estructuralismo, un hecho social total no refleja necesariamente estructuras sociales inmutables. El chancho y el cuy sufren cambios porque viven en una sociedad en transformación. El caso amantaneño confirma que el habitus sobre la higiene depende de la transformación en la concepción de lo que es limpio y de lo que es sucio, de la pureza y de la polución. Como hemos visto, es resultado de una conjunción de vectores que hemos categorizado en dos tipos: aquellos que, de forma explícita o implícita, buscan disciplinar al indígena rural (turismo, escolarización, religión); y aquellos que han facilitado la propagación por contagio de costumbres del mundo urbano y mestizo (emigración, doble residencia, nuevas tecnologías de la comunicación).

Pero la activación práctica de la nueva concepción de limpieza no habría sido posible sin cambios en el contexto económico y en las estrategias de reproducción del amantaneño. Estos cambios le 
permitieron abandonar aquellas praxis, en el sentido bourdieano del término, que ahora estaban en desacuerdo con la nueva concepción de limpieza, higiene y pureza. La desaparición del chancho, víctima de la conjunción de diferentes variables, de una tormenta perfecta, es un ejemplo emblemático. La cita con la que hemos abierto esta sección procede de un isleño que, en la época en que lo expresó (1990), era un fervoroso adventista. Su hermano, al que alude, también. Aún más: era uno de los líderes de la congregación. Recodemos que para el adventismo, chanchos y cuyes entran en la categoría de animales impuros. Impuros en un doble sentido: espiritual y físico. Por tanto, no solo la ingesta es problemática; la proximidad también contamina. Sin embargo, a principios de la década de 1990, un adventismo pasado por el tamiz de Amantaní permitía criar estos animales si se destinaban a su venta y no al autoconsumo.

Esto evidencia la segunda tesis de nuestro trabajo: la asunción de los cambios en la percepción de limpieza y la adquisición de nuevas prácticas higiénicas no necesariamente tienen lugar a la vez. En Amantaní hubo una divergencia temporal. El cambio en la mentalidad fue anterior al cambio en las prácticas; el isleño solo las activó cuando se encontró en el contexto material adecuado. En otras palabras, la transformación del habitus como principio generador de prácticas no siempre es coetánea con la transformación del habitus como principio generador de representaciones. Los cambios en el habitus no son necesariamente un proceso sincopado y uniforme. En Amantaní, la representación de lo que se entiende por limpieza y pureza fue anterior a la adopción de prácticas coherentes con la nueva concepción. Solo cuando se dieron las condiciones materiales apropiadas se pudieron activar. Mientras esto no fue posible, la sociedad tuvo que lidiar con la incongruencia mirando hacia otro lado o, como en el caso del chancho y los creyentes adventistas, creando un discurso autóctono que lo justificase. No sería hasta principios de la década de 2010 que $\mathrm{H}$ y su hermano dejaron de criar cuyes y cerdos. Curiosamente, por entonces hacía años que habían abandonado la congregación adventista por diferentes motivos. La impronta, no obstante, había quedado.

Además, los ritmos en el cambio en la apreciación de la limpieza y la higiene, y en la asunción de nuevas prácticas, no fue homogénea en toda la sociedad amantaneña. Factores como la edad, la participación en la actividad turística o el grado de relación con el mundo exterior, jugaron un papel. Ya hemos visto como a los más ancianos se les limita su acceso a los beneficios del turismo aduciendo que no pueden ofrecer un servicio de calidad; calidad turística incluye, entre otras cosas, una concepción de la higiene urbana y occidental. Los últimos isleños que criaron chanchos fueron familias que vivían a cierta distancia de los núcleos más poblados y no alojaban turistas. Aquellos que pasaban más tiempo en la emigración, o que empezaron a tener una doble residencia, difícilmente podían mantener un chancho que requiere cuidados a diario.

La sociedad rural e indígena se ocupa de la limpieza, tanto material como simbólicamente, de forma diferente a como lo hace la urbana y criolla (Moser 2007). Pero también es cierto que la relación inequitativa y de subordinación que hay entre estos dos mundos hace que las segundas tiendan a transferir sus valores a las primeras, como vasos comunicantes situados a diferente nivel. Esta transferencia no ha sido automática, ni escrupulosa. El amantaneño ha asumido los principios urbano-criollos sobre limpieza y pureza a su manera y ritmo. Y es posible que, si las condiciones materiales cambian, también cambien sus prácticas al respecto. No olvidemos que el campesino adecua su modelo de producción a las condiciones del mercado. Si estas condiciones empeoran, tiende a aplicar estrategias dirigidas a conseguir una mayor autonomía económica (Ploeg 2012). En este caso, podría readmitir al chancho en sus vidas, y concebir una nueva percepción acorde sobre limpieza e higiene.

Agradecimientos: El presente trabajo forma parte de una investigación más amplia que ha sido posible gracias al apoyo del proyecto "Turismo de base local y resiliencia socio-ecológica", otorgado por el Ministerio de Economía, Industria y Competitividad del Estado Español (Ref. CSO2017-84893-P). El autor agradece a los evaluadores sus útiles comentarios, que han permitido mejorar el artículo en las diferentes revisiones.

\section{Referencias Citadas}

Asensio, R.H. 2017. Los Nuevos Incas: La Economía Política del Desarrollo Rural Andino en Quispicanchi (2000-2010). Instituto de Estudios Peruanos, Lima.

Ávalos de Matos, R. 1951. Changements culturels dans les Iles du lac Titicaca. Travaux de l'Institut Francais d'Etudes Andines 3:40-50.
Bourdieu, P. 1979. La Distinction: Critique Sociale du Jugement. Les Éditions de Minuit, Paris.

Bourdieu, P. 1980. Le Sens Pratique. Les Éditions de Minuit, Paris.

Cazeneuve, J. 1968. Sociologie de Marcel Mauss. Presses Universitaires de France, Paris. 
Colloredo-Mansfeld, R. 1998. 'Dirty Indians', radical indígenas, and the political economy of social difference in modern Ecuador. Bulletin of Latin American Research 17 (2):185-205.

Degregori, C.I. 2003. Perú: identidad, nación y diversidad cultural. En Territorio, Cultura e Historia: Materiales para la Renovación de la Enseñanza sobre la Sociedad Peruana, editado por P. Oliart, pp. 212-228. GTZ (Cooperación Técnica Alemana), PromPerú e Instituto de Estudios Peruanos, Lima.

Douglas, M. 2003 [1966]. Purity and Danger: AnAnalysis of Concepts of Pollution and Taboo. Routledge, London.

Fanon, F. 2015 [1952]. Peau Noire, Masques Blancs. Seuil, Paris.

Fuenzalida, F., J. Golte, T. Valiente y J.L. Villarán1982. El Desafio de Huayopampa: Comuneros y Empresarios. Instituto de Estudios Peruanos, Lima.

Gascón, J. 1996. La polémica sobre la tragedia de los comunes: Un caso andino. Debate Agario 25:21-35.

Gascón, J. 1999. El control y explotación de la mano de obra colona en la hacienda andina peruana. Anuario de Estudios Americanos $56(1): 195-215$.

Gascón, J. 2005a. Gringos como en sueños: Diferenciación y Conflicto Campesino en los Andes Peruanos ante el Desarrollo del Turismo. Instituto de Estudios Peruanos, Lima.

Gascón, J. 2005b. Coerción sorda de las relaciones económicas: Aplicación de un concepto marxista para comprender las relaciones de producción en la hacienda andina. Sociedad y Economía 8:59-110.

Gascón, J. 2011. Turismo rural y diferenciación campesina: Consideraciones a partir de un caso andino. Mundo Agrario 22:1-18.

Gascón, J. 2017. Acabando con la administración de poblaciones: Razones y estrategias para reclamar la plena ciudadanía en la hacienda surandina peruana. En Poderes y Personas: Pasado y Presente de la Administración de Poblaciones en América Latina, editado por V. Bretón y M.J. Vilalta, pp. 195-216. Icaria, Barcelona.

Gascón, J. y M. Martínez Mauri 2017. Isleños y turistas: Propiedad comunitaria y territorialidad en sociedades indígenas. Gazeta de Antropología 33 (1):08.

Golte, J. 1980. La Racionalidad de la Organización Andina. Instituto de Estudios Peruanos, Lima.

Gonzales de Olarte, E. 1994. En las Fronteras del Mercado: Economía Política del Campesinado en el Perú. IEP, Lima.

Gurvitch, G. 1990 [1964]. Varieties of Social-time. En The Sociology of Time, editado por J. Hassard, pp. 67-76. Palgrave Macmillan, New York.

Instituto Nacional de Estadística e Informática (INEI) 2018. Censos Nacionales 2017: XII de Población, VII de Vivienda y III de Comunidades Indígenas. Tomo I: Puno. INEI, Lima.

Kingman, E. y V. Bretón 2017. Las fronteras arbitrarias y difusas entre lo urbano-moderno y lo rural-tradicional en los Andes. The Journal of Latin American and Caribbean Anthropology 22 (2):235-253.

Lévi-Strauss, C. 1950. Introduction à l'oeuvre de M. Mauss. En Sociologie et Anthropologie, de M. Mauss. Presses Universitaires de France, Paris.

Mauss, M. 2009 [1925]. Ensayo sobre el Don: Forma y Función del Intercambio en las Sociedades Arcaicas. Katz, Buenos Aires.
MINCETUR (Ministerio de Comercio Exterior y Turismo) 2019a. Llegada de visitantes a la Isla Amantani del Lago Titicaca (18/8/2019). Dirección http://datosturismo.mincetur.gob.pe/appdatosTurismo/ Content2.html

MINCETUR (Ministerio de Comercio Exterior y Turismo) 2019b. Estadísticas de turismo: Flujo de turistas internacionales e ingreso de divisas por turismo receptivo (18/8/2019). http://datosturismo. mincetur.gob.pe/appdatosTurismo/Content1.html

Moser, W. 2002. The acculturation of waste. En Waste-Site Stories: The Recycling of Memory, editado por B. Neville y J. Villeneuve, pp. 85-105. The State University of New York Press, Albany.

Moser, W. 2007. Garbage and recycling: From literary theme to mode of production. Other Voices 3 (1):83-103.

Orlove, B.S. 1998. Down to earth: race and substance in the Andes. Bulletin of Latin American Research 17 (2):207-222.

Pérez, L.M, I. Delgadillo y D.P. García 2014. Alteridad e identidad: dos categorías en tensión para comprender el pensamiento de niños, niñas y adolescentes colombianos sobre lo indígena. Infancias Imágenes 13 (1):90-101.

Perche, G. 2012. Cuando el cuy tuvo que salir de la cocina: turismo e intimidade na ilha de Amantani, Lago Titicaca, Perú. En ¿El turismo es Cosa de Pobres? Patrimonio Cultural, Pueblos Indígenas y Nuevas Formas de Turismo en América Latina, editado por R.H. Asensio y B. Pérez Galán, pp. 201-224. Pasos, Instituto de Estudios Peruanos, Tenerife.

Ploeg, J.D. van der 2012. The New Peasantries: Struggles for Autonomy and Sustainability in an Era of Empire and Globalization. Routledge, London.

Ramos, D.D. 2008. Caracterización de la Canal y la Carne del Cerdo Criollo y de los Productos Cárnicos en el Departamento de Tumbes, Perú. Tesis Doctoral, Departamento de Higiene y Tecnología de los Alimentos, Universidad de León, León.

Reno, J. 2015. Waste and waste management. Annual Review of Anthropology 44:557-572.

Ríos Ocsa, B. 1992. Ganadería y Economía Campesina: La Importancia de los Animales en los Sistemas Tradicionales de Producción en la Sierra Sur de Cusco. Centro Bartolomé de Las Casas, Cusco.

Smith, G.A. 1978. Socio-economic differentiation and relations of production among Rural-based petty producers in Central Peru, 1880 to 1970. The Journal of Peasants Studies 6 (3):287-310.

Smith, V. 2007. Clean: A History of Personal Hygiene and Purity. Oxford University Press, Oxford.

Swanson, K. 2007. Revanchist urbanism heads south: the regulation of indigenous beggars and street vendors in Ecuador. Antipode 39 (4):708-728.

Swanson, K. 2010. Pidiendo Caridad en la Ciudad: Mujeres y Niños Indígenas en las Calles del Ecuador. FLACSO y Abya-Yala, Quito.

Toche, E. 2005. Servicio militar y la construcción nacional: Notas sobre el origen de la institución. Investigaciones Sociales 9 (14):395-409.

Urrutia, J., M.I. Remy y M.L Burneo 2019. Comunidades Campesinas y Nativas en el Contexto Neoliberal Peruano: Una Lectura del CENAGRO y las Hojas de Información Complementarias. Instituto de Estudios Peruanos y Centro Peruano de Estudios Sociales, Lima. 


\section{Notas}

1 Entre estos pocos trabajos, hemos de destacar el de ColloredoMansfeld (1998), que analiza el estereotipo del indígena como individuo sucio, el de Perche (2012), que relaciona los cambios en la percepción de la limpieza por influencia del turismo, y los de Swanson $(2007,2010)$, sobre las políticas contra los mendigos indígenas en nombre de un proyecto higienista.

2 La población de Amantaní ha pasado de unos 1.700 habitantes a mediados del siglo XX (Ávalos de Matos 1951) a unos 3.500 a finales de la década de 2010 (dato de la Municipalidad).

3 Esta misma fuente estadística indica que al año siguiente, en 2018, el número de visitantes disminuyó a 25.561. Los isleños y la Municipalidad de Amantaní niegan esta disminución

drástica. Por el contrario, afirman que el número de turistas no ha dejado de aumentar desde la década de 1990.

4 Hemos analizado la distribución de los beneficios del turismo en Amantaní en diversos trabajos (p.ej., Gascón 2005a, 2011; Gascón y Martínez Mauri 2017; Pérez Berenguer y Gascón 1997). La desigual distribución de los beneficios de los comunes en el Perú andino ha sido estudiada desde la década de 1980 (p.ej., Fuenzalida 1982; Golte 1980; Gonzales de Olarte 1984; Gascón 1996; Smith 1978; Urrutia et al. 2019)

5 Hemos estudiado este proceso en diversos trabajos (Gascón 1999, 2005b, 2017). 
